\title{
Physical Modeling of SiC Power Diodes with Empirical Approximation
}

\author{
Leobardo Hernández ${ }^{\dagger}$, Abraham Claudio*, Marco A. Rodríguez ${ }^{* *}$, Mario Ponce*, and Alejandro Tapia*** \\ $\dagger * * *$ Departamento de Ingeniería en Comunicaciones y Electrónica del I.P.N., Mexico City, México \\ * Centro Nacional de Investigación y Desarrollo Tecnológico (CENIDET) Cuernavaca, Morelos, México \\ ** Universidad Autónoma del Carmen (UNACAR), Ciudad del Carmen, Campeche, México
}

\begin{abstract}
This article presents the development of a model for $\mathrm{SiC}$ power diodes based on the physics of the semiconductor. The model is able to simulate the behavior of the dynamics of the charges in the $\mathrm{N}$ - region based on the stored charge inside the $\mathrm{SiC}$ power diode, depending on the working regime of the device (turn-on, on-state, and turn-off). The optimal individual calculation of the ambipolar diffusion length for every phase of commutation allows for solving the ambipolar diffusion equation $(A D E)$ using a very simple approach. By means of this methodology development a set of differential equations that models the main physical phenomena associated with the semiconductor power device are obtained. The model is developed in Pspice with acceptable simulation times and without convergence problems during its implementation.
\end{abstract}

Key Words: Physical Modeling, Silicon Carbide, Simulation Semiconductor

\section{INTRODUCTION}

Silicon carbide $(\mathrm{SiC})$ is an attractive material for replacing the silicon used in the manufacturing of power devices. SiC presents better properties than silicon, such as: a wide bandgap $(2 \mathrm{x})$, a higher saturation electron drift velocity $(2 \mathrm{x})$, a high thermal conductivity $(5 \mathrm{x})$ and a very high breakdown field (10x) [1]-[3]. Among the different silicon carbide power devices that have been developed [4]-[11], the $\mathrm{SiC}$ power diode structure is one of most interesting. It can appear as a discrete component or as an internal diode in more complex power conversion circuits. Diverse modeling methodologies have appeared in the literature for the development of models for semiconductor power devices. According to [12] these can be classified in two groups: functional models and analytical solutions. The proposed approaches in the last group can be subdivided into: separation of variables, transformation (Laplace, Fourier), concentrated charges and numerical solutions [13]-[16]. This type of methodology is known as a physical model. It is one of most efficient and it is mainly concerned with modeling the charges in the $\mathrm{N}$ - region, where every physical phenomenon of the device is related to a

Manuscript received Dec. 23, 2010; revised Feb. 25, 2011

Recommended for publication by Associate Editor Chong-Man Yun.

$\dagger$ Corresponding Author: 1hernandezg@ipn.mx, bilbito_98@yahoo.com Tel: +55-562-420-00, ESIME-Culhuacan

* Centro Nacional de Investigación y Desarrollo Tecnológico (CENIDET) Cuemavaca, México

** Universidad Autónoma del Carmen (UNACAR), Ciudad del Carmen, México

*** Departamento de Ingeniera en Comunicaciones y Electrónica del I.P.N., México specific term in a set of derived equations.

The proposed model describes the static and dynamic behaviors with a temperature dependency in the experimental operation range $\left(25^{\circ} \mathrm{C}-225^{\circ} \mathrm{C}\right)$ for silicon carbide, which has been reported in the literature [13]-[15]. Furthermore, obtaining a set of linear differential equations allows simulating in Pspice the main associate physical phenomena for the semiconductor device, as well as modeling of the injection and the evacuation of charges in the $N$ - region. The proposed methodology solves the $A D E$ with dependency on the adjustment of the ambipolar diffusion length $(L)$, which is achieved by using a simplified semi-theoretical approach. For every commutation phase, the proposed suitable analytical expression allows for the calculation of a new $L$ value.

\section{Modeling PRINCIPLE}

The power diode structure has been simplified in order to help the mathematical analysis. As a result, only two conventional $p-n$ junctions are supposed. Fig. 1(a) shows the typical structure of the power diode which is used for the physical design of this kind of device. Fig. 1(b) shows the simplified structure used to model the $\mathrm{SiC}$ power diode.

Fig. 2 shows the basic structure of a power diode. In this Figure, $I_{n 0}, I_{p 0}, I_{n W}$ and $I_{p W}$, represent the injected currents into the $N$ - region, $V_{J 1}$ and $V_{J 2}$ represent the drop voltages of the $P^{+} N^{-}$and $N^{-} N^{+}$junctions, respectively, $x_{L}$ and $x_{R}$ represent the limits of the depletion regions within $N-, W^{\prime}$ represents the width of the depletion region formed in the $P^{+} N^{-}$junction, and $W=W_{B}-W^{\prime}$ is the effective width of the $N-$ region. 


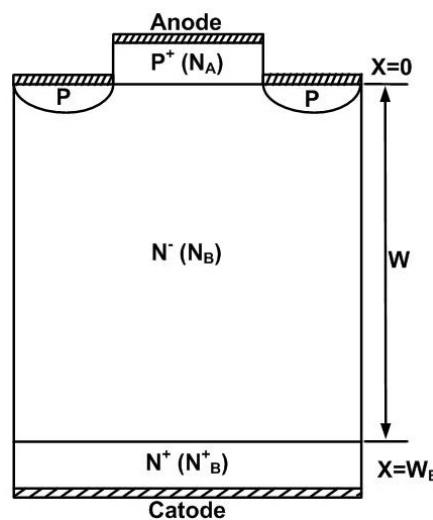

(a)

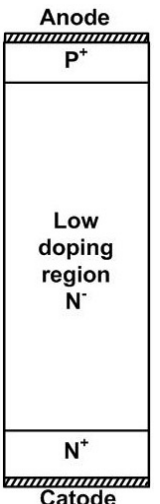

(b)
Fig. 1. Structure for: (a) Design, (b) Modeling.

Assuming a high-level injection, the charge dynamics are described by the ambipolar diffusion equation (1):

$$
\frac{\partial^{2} p(x, t)}{\partial x^{2}}=\frac{p(x, t)}{L^{2}}+\frac{1}{D} \frac{\partial p(x, t)}{\partial t} .
$$

The currents depicted in the Figure 2, can be calculated from the transport equations given by (2) and (3), and the total $N-$ region charge, $Q_{B}$, is expressed by (4) [17], [18].

$$
\begin{aligned}
I_{n(x, t)} & =\frac{b \cdot I_{T}}{1+b}+q A D \cdot \frac{\partial p(x, t)}{\partial x} \\
I_{p(x, t)} & =\frac{I_{T}}{1+b}-q A D \cdot \frac{\partial p(x, t)}{\partial x} \\
Q_{B} & =q A \cdot \int_{0}^{W} p(x, t) \cdot d x .
\end{aligned}
$$

In a high injection condition, the low resistance that appears in the $N$ - region is the result of a high level concentration of charges that are injected by both emitters and it allows for the transport of a high current through a power diode with a low on-state voltage drop. The value of the resistance $R_{M}$, in the $N$ - region for the semiconductor power devices is given by (5), [17], [18].

$$
R_{M}=\int_{0}^{W} \frac{d x}{q A \cdot\left(\mu_{n} n+\mu_{p} p\right)}=\frac{W^{2}}{\left(\mu_{n}+\mu_{p}\right) \cdot Q_{B}+\mu_{n} Q_{0}}
$$

where $Q_{0}$ represents the charge stored in the thermodynamicequilibrium. To calculate the $V_{J 1}$ and $V_{J 2}$ voltages, the Shockley equation will be used in (6) and (7). The mathematical expressions (1)-(7) are the basis of the static and dynamic models of the $\mathrm{SiC}$ power diode.

$$
\begin{aligned}
& I_{n 0}=I_{P^{+} S}\left[\exp \left(\frac{V_{j 1}}{V_{T}}\right)-1\right] \\
& I_{n W}=I_{N^{+} S}\left[\exp \left(\frac{V_{j 2}}{V_{T}}\right)-1\right]
\end{aligned}
$$

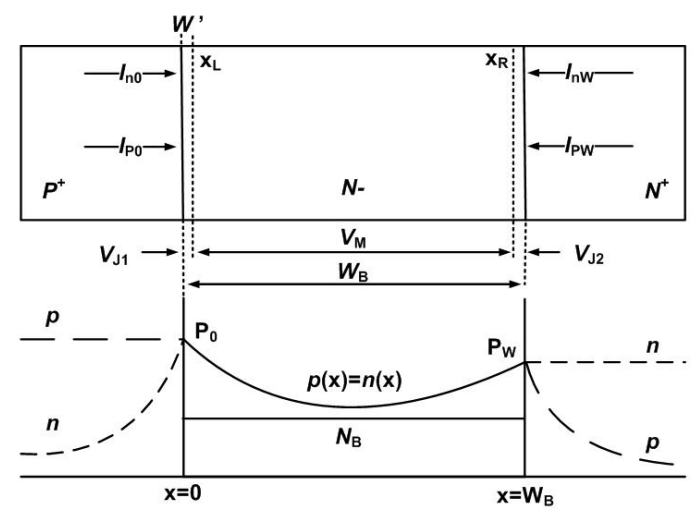

Fig. 2. Power diode basic structure and charges distribution.

\section{STATIC STATE MODEL}

\section{A. Carrier distribution $p(x)$}

Equation (1) can be reduced to a differential equation. This is because during the on-state condition, $\partial p(x, t) / \partial t=0$. Under this consideration the analytical solution of (1) is given by (8).

$$
p(x)=\frac{P_{W} \cdot \operatorname{Sinh}\left(\frac{x}{L_{S}}\right)+P_{0} \cdot \operatorname{Sinh}\left(\frac{W-x}{L_{S}}\right)}{\operatorname{Sinh}\left(\frac{W}{L_{S}}\right)}
$$

where $L_{S}=\sqrt{D \cdot \tau}$ represents the on-state ambipolar diffusion length, where $\tau$ is the steady state life time defined in [13]. $P_{0}$ and $P_{W}$ represent the carrier concentrations at the two edges of the $N$ - region. The boundary concentrations $P_{0}$ and $P_{W}$ are calculated under the quasi-equilibrium condition for a highlevel injection condition [19].

$$
\begin{aligned}
P_{0} & =n_{i} \sqrt{\frac{I_{n 0}}{I_{P^{+} S}}} \\
P_{W} & =n_{i} \sqrt{\frac{I_{n W}}{I_{N^{+} S}}}
\end{aligned}
$$

where $I_{P^{+} S}$ and $I_{N^{+} S}$ represent the saturation current in the $P^{+} N^{-}$and $N^{-} N^{+}$junctions, respectively. $I_{n 0}$ and $I_{n W}$ can be calculated from (6) and (7). The stored charges $Q_{B}$ in the $N^{-}$region can be calculated by (11), which is obtained by substituting (8) into (4).

$$
Q_{B}=q A L_{S} \cdot\left(P_{0}+P_{W}\right) \operatorname{Tanh}\left(\frac{W}{2 L_{S}}\right) .
$$

That allows the calculation of the modulation resistance according to (5), which is obtained as follows:

$$
R_{M}=\frac{W^{2}}{\left[\mu_{n}+\mu_{p}\right]\left[q A L_{S}\left(P_{0}+P_{W}\right) \operatorname{Tanh}\left(\frac{W}{2 L_{S}}\right)\right]+\mu_{n} Q_{0}} .
$$

\section{B. Modeling the injected currents}

The total injected current is the sum of the hole and electron currents. The Pspice implementation is performed because the electron currents are modeled through standard diodes adjusted to the SiC electrical characteristics, Equ.(6) and (7), and the 


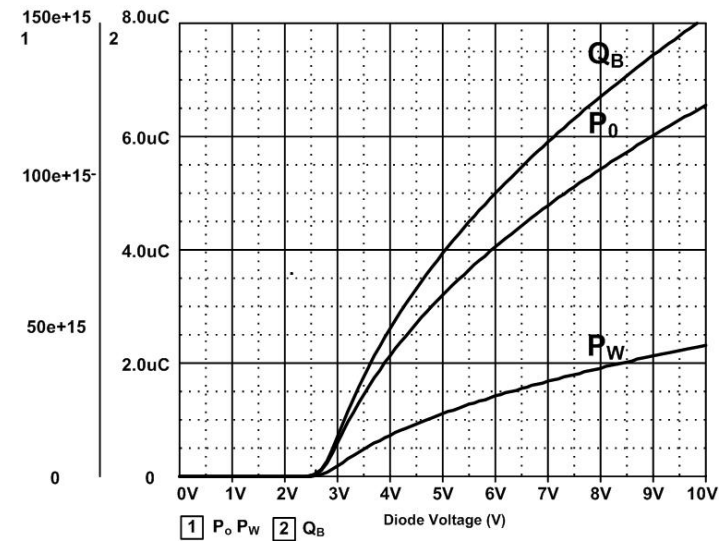

Fig. 3. Simulation results for the $P_{0}, P_{W}$, and $Q_{B}$ with $V_{O N} \approx 2.8 \mathrm{~V}$.

holes currents are modeled by the transport equation, Equ. (13) and (14).

$$
\begin{gathered}
I_{p 0}=\frac{I_{T}}{1+b}-\left.q A D \cdot \frac{\partial p(x)}{\partial x}\right|_{x=0} \\
I_{p W}=\frac{I_{T}}{1+b}-\left.q A D \cdot \frac{\partial p(x)}{\partial x}\right|_{x=W}
\end{gathered}
$$

The gradients of the carrier concentrations are obtained from (8), which is evaluated at $x=0$ and $x=W_{B}$.

$$
\begin{aligned}
& \left.\frac{d p(x)}{d x}\right|_{x=0}=\frac{1}{L_{S}} \frac{P_{W}-P_{0} \cdot \operatorname{Cosh}\left(\frac{W}{L_{S}}\right)}{\operatorname{Sinh}\left(\frac{W}{L_{S}}\right)} \\
& \left.\frac{d p(x)}{d x}\right|_{x=W}=\frac{1}{L_{S}} \frac{P_{W} \cdot \operatorname{Cosh}\left(\frac{W}{L_{S}}\right)-P_{0}}{\operatorname{Sinh}\left(\frac{W}{L_{S}}\right)}
\end{aligned}
$$

In Fig. 3, the simulation results for $P_{0}, P_{W}$ and $Q_{B}$ are presented. For $n_{i} \approx 6.7 \times 10^{-11} \mathrm{~cm}^{-3}$ and assuming that the $N_{A} \cdot N_{D}$ product has the typical value of $10^{35} \mathrm{~cm}^{-6}$, the theoretically expected on-state voltage is $2.70 \mathrm{~V}$ [17], [18]. The obtained simulation results concerning the on-state voltage are corroborated by the expected theoretical value, which is $2.8 \mathrm{~V}$, as can be seen in Fig. 3 .

\section{DYNAMIC MODEL}

\section{A. Turn-on carrier distribution $p(x, t)$ solution}

The solution of $p(x, t)$ is similar to the on-state case. An interesting contribution has been introduced in the proposed dynamic model which is dependent on time based on a new $L_{O N}$, as can be observed in (17). The $L_{O N}(x, t)$ variation is based on the stored charges $Q_{B}$, which are installed in the $N-$ region depending on time, during this commutation.

$$
p(x, t)=\frac{P_{W} \cdot \operatorname{Sinh}\left(\frac{x}{L_{O N}(x, t)}\right)+P_{0} \cdot \operatorname{Sinh}\left(\frac{W-x}{L_{O N}(x, t)}\right)}{\operatorname{Sinh}\left(\frac{W}{L_{O N}(x, t)}\right)} .
$$

The holes currents are modeled by the transport equation again, equations (13) and (14) with (17). The new gradients evaluated in $x=0$ and $x=W$, as well as the calculation of the $Q_{B O N}$ charges that are injected during the turn-on, are presented in (18)-(20).

$$
\begin{gathered}
\left.\frac{d p(x, t)}{d x}\right|_{x=0}=\frac{P_{W}-P_{0} \cdot \operatorname{Cosh}\left(\frac{W}{L_{O N}(x, t)}\right)}{L_{O N}(x, t) \cdot \operatorname{Sinh}\left(\frac{W}{L_{O N}(x, t)}\right)} \\
\left.\frac{d p(x, t)}{d x}\right|_{x=W}=\frac{P_{W} \cdot \operatorname{Cosh}\left(\frac{W}{L_{O N}(x, t)}\right)-P_{0}}{L_{O N}(x, t) \cdot \operatorname{Sinh}\left(\frac{W}{L_{O N}(x, t)}\right)} \\
Q_{B_{O N}}=q A L_{O N}(x, t) \cdot\left(P_{0}+P_{W}\right) \operatorname{Tanh}\left(\frac{W}{2 L_{O N}(x, t)}\right) .
\end{gathered}
$$

\section{B. Diffusion length $\operatorname{LON}(x, t)$ calculation}

To calculate $L_{O N}(x, t)$, the charge control equation (21) is modified by the introduction of a new stored charge variable, $Q_{B}=Q_{B O N}$.

$$
I_{T}=\frac{Q_{B_{O N}}}{\tau}+\frac{d Q_{B_{O N}}}{d t}
$$

Under the conditions where $I_{p 0}>I_{n 0}$ then $I_{T} \approx I_{p 0}-I_{p W}$ and by replacing the previous conditions into (21) the following modified expression is obtained:

$$
I_{p 0}-I_{p W}=\frac{Q_{B_{O N}}}{\tau}+\frac{d Q_{B_{O N}}}{d t} .
$$

Combining (13), (14), (18), (19) and (20) with (22), the final expression used to calculate $L_{O N}(x, t)$ is obtained.

$$
L_{O N}(x, t)=\sqrt{\frac{D \cdot Q_{B_{O N}}}{\frac{Q_{B_{O N}}}{\tau}+\frac{d Q_{B N}}{d t}}} .
$$

Analyzing (23), it is possible to conclude that the expected theoretical behavior is as follows:

- Before the beginning the injection of currents to the $N$ region, the stored charges are $\left(Q_{B O N}=0\right)$ and $L_{O N}(x, t)=$ $L_{S}$. It can be concluded that the power diode structure behavior is under a static condition.

- When the injection begins $\left(Q_{B O N}>0\right)$, a high injection of charges is imposed $\left(d Q_{B_{O N}} / d t>0\right)$, which implies $L_{O N}(x, t)<L_{S}$, as can be seen in (23).

- During the turn-on, the injected charges reach the maximum value $\left(Q_{B O N}=Q_{B}\right)$, and are stabilized for the onstate condition, which implies $L_{O N}(x, t)=L_{S}$.

Fig. 4 shows the simulation results for the $L_{O N}(x, t)$ variation during the turn-on. During this phase, $L_{O N}(x, t)$ takes the minimum value of $0.597 \times 10^{-3} \mathrm{~cm}$ and converges to the static value of $L_{S}=0.737 \times 10^{-3} \mathrm{~cm}$. A simulation of the injected charges, $Q_{B}$, is also observed, where $Q_{0}=104 \mathrm{nC}$. At $t \approx 20.20 \mathrm{nS}$ the turn-on ends and the on-state initiates. Based on the simulation results, the validity of the $L_{O N}(x, t)$ expression to solve $p(x)$ is verified. 


\section{Turn-off carrier distribution $p(x, t)$ solution}

Fig. 5 shows the typical reverse recovery waveforms of current and voltage that are observed in typical applications of power converters. The typical carrier distributions evolution, during this phase, can be observed in the numerical results shown in Fig. 6 [20]. In order to obtain an adequate $p(x, t)$ approximation, it is proposed to model the charges, by modelling the three sections defined by a typical distribution for this kind of devices. This means a hyperbolic distribution of the central part, and a sinusoidal distribution of the lateral sides. It can be observed in Fig. 7, where $P_{L}$ and $P_{R}$ represent the initial and final concentrations for the $p_{2(x)}$ section. The proposed expressions to model every section are observed in (25)-(27).

$$
\begin{gathered}
p_{1(x, t)}=P_{L} \cdot \operatorname{Cos}\left(\frac{x-x_{L}}{L_{L}(x, t)}\right) \\
p_{2(x, t)}=\frac{P_{R} \cdot \sinh \left(\frac{x-x_{L}}{L_{M}(x, t)}\right)+P_{L} \cdot \sinh \left(\frac{x_{R}-x}{L_{M}(x, t)}\right)}{\sinh \left(\frac{x_{R}-x_{L}}{L_{M}(x, t)}\right)} \\
p_{3(x, t)}=P_{R} \cdot \operatorname{Cos}\left(\frac{x-x_{R}}{L_{R}(x, t)}\right) .
\end{gathered}
$$

The three new diffusion lengths $\left(L_{L}(x, t), L_{M}(x, t)\right.$ and $\left.L_{R}(x, t)\right)$ are very important due to the fact that they model the curvature in every section for simulation. The new ambipolar diffusion lengths for every section are obtained as follows: for $L_{L}(\mathrm{x}, \mathrm{t})$, the $p_{1}(x, t)$ derivative evaluated in $x=0$, is:

$$
\left.\frac{\partial p_{1(x, t)}}{\partial x}\right|_{x=0}=\frac{P_{L}}{L_{L}(x, t)} \cdot \sin \left(\frac{x_{L}}{L_{L}(x, t)}\right) .
$$

Replacing in (27), the $P_{0} / P_{L}$ evaluated at $x=0$ from (24) and using the identity $\cos ^{-1}(x)=\sin ^{-1} \sqrt{1-x^{2}}$, the final expression for $L_{L}(x, t)$ is given in (28).

$$
L_{L}(x, t)=\frac{\sqrt{P_{L}^{2}-P_{0}^{2}}}{\left.\frac{d p(x, t)}{d x}\right|_{x=0}} .
$$

For $L_{M}$, the total current is used. It can be approximated to the turn-on analogously.

$$
I_{T(x)} \approx I_{p\left(x_{L}\right)}-I_{p\left(x_{R}\right)} .
$$

Replacing (29) into (22) and considering the displacement currents that appear in the depletion regions, the following is obtained:

$$
I_{p\left(x_{L}, t\right)}-I_{p\left(x_{R}, t\right)}=\frac{Q_{M}}{\tau}+\frac{d Q_{M}}{d t}-A q P_{L} \frac{d x_{L}}{d t}+A q P_{R} \frac{d x_{R}}{d t} .
$$

To calculate $I_{p(x L, t)}$ and $I_{p(x R, t)}$, (3) is evaluated in $x_{L}$ and $x_{R}$. Replacing this into (30), the final expression for $L_{M}$ is obtained.

$$
L_{M}(x, t)=\sqrt{\frac{D \cdot Q_{M}}{\frac{Q_{M}}{\tau}+\frac{d Q_{M}}{d t}-q A P_{L} \frac{d x_{L}}{d t}+q A P_{R} \frac{d x_{R}}{d t}}} .
$$

For $L_{R}(x, t)$, the $p_{3}(x, t)$ derivative evaluated in $x=W$ is obtained.

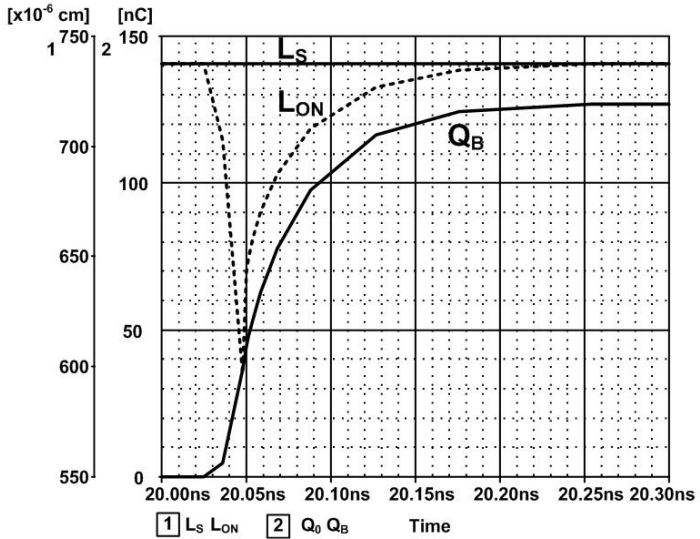

Fig. 4. $L_{O N}(x, t)$, and $Q_{B}$ behavior in relation to $L_{S}$ y $Q_{0}$ respectively.

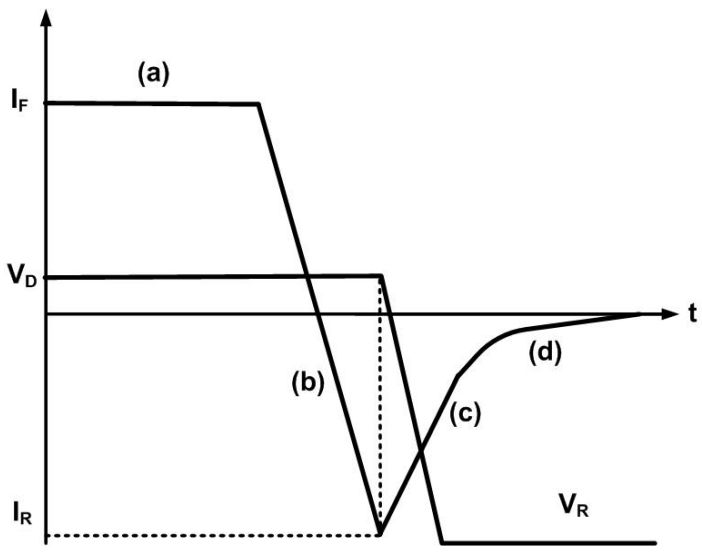

Fig. 5. Reverse recovery waveforms for power diode.

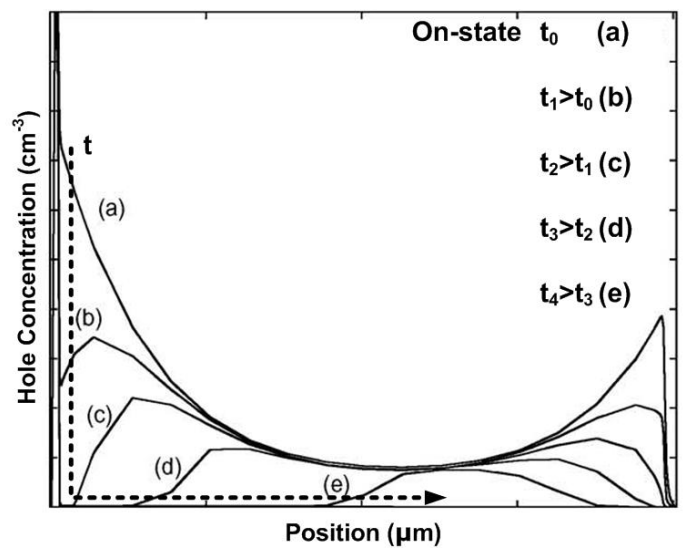

Fig. 6. $p(x, t)$ numerical results during the turn-off phase.

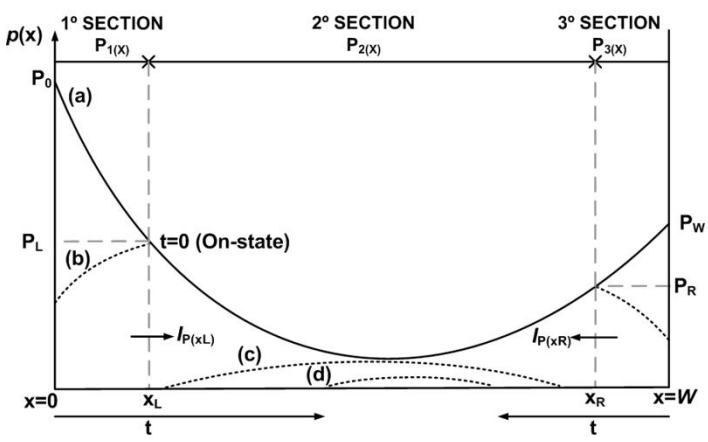

Fig. 7. $p(x)$ modeling proposal for the turn-off phase. 


$$
\left.\frac{\partial p_{3(x, t)}}{\partial x}\right|_{x=W}=\frac{P_{R}}{L_{R}(x, t)} \cdot \sin \left(\frac{W-x_{R}}{L_{R}(x, t)}\right) .
$$

Replacing in (32), the $P_{W} / P_{R}$ relation evaluated in $x=W$ of (26) and using the identity $\cos ^{-1}(x)=\sin ^{-1} \sqrt{1-x^{2}}$, the $L_{R}(x, t)$ final expression is obtained.

$$
L_{R}(x, t)=\frac{\sqrt{P_{R}^{2}-P_{W}^{2}}}{\frac{d p(x, t)}{d x} \mid x=W} .
$$

As in Equ. (23) during the on-state, the development expressions given by (28), (31) and (33) can allow modeling the time dependency in a continuous way, which can guarantee an adequate approximation of the model. The new gradients for $p(x, t)$ evaluated in $x=0, x_{L}, x_{R}$ and $x=W$, are calculated as follows: for the $0<x \leq x_{L}$ range, it is fulfilled:

$$
I_{p(0, t)}-I_{p\left(x_{L}, t\right)}=\frac{Q_{L}}{\tau}+\frac{d Q_{L}}{d t}-A q P_{L} \cdot \frac{d x_{L}}{d t} .
$$

$I_{p(0)}, I_{p\left(x_{L}\right)}$, are calculated by (3) evaluated in $x=x_{L}$ and $x=$ 0 , as $d p\left(x=x_{L}\right) / d x=0$. By replacing the previous conditions into (34), the following is obtained:

$$
\left.\frac{d p(x, t)}{d x}\right|_{x=0}=\frac{1}{q A D}\left[-\frac{Q_{L}}{\tau}-\frac{d Q_{L}}{d t}+q A P_{L} \cdot \frac{d x_{L}}{d t}\right]
$$

For the $x_{L} \leq x \leq x_{R}$ range, the following is fulfilled:

$$
I_{p\left(x_{R}, t\right)}-I_{p(W, t)}=\frac{Q_{R}}{\tau}+\frac{d Q_{R}}{d t}-A q P_{R} \cdot \frac{d x_{R}}{d t} .
$$

$I_{p\left(x_{R}, t\right)}, I_{p(W, t)}$ are calculated with (3) evaluated in $x=x_{R}$ y $x=W$, as $d p\left(x=x_{R}\right) / d x=0$. By the substitution of these conditions into (36), the following is obtained:

$$
\left.\frac{d p(x, t)}{d x}\right|_{x=W}=\frac{1}{q A D}\left[\frac{Q_{R}}{\tau}-\frac{d Q_{R}}{d t}-q A P_{R} \cdot \frac{d x_{R}}{d t}\right] .
$$

The expressions for calculating $Q_{L}, Q_{M}$ and $Q_{R}$, are obtained by evaluating the $p(x, t)$ integral for every proposed section. This is given by (38), (39) and (40).

$$
\begin{gathered}
Q_{L}=q A L_{L}(x, t) \sqrt{P_{L}^{2}-P_{0}^{2}} \\
Q_{M}=q A L_{M}(x, t)\left(P_{R}+P_{L}\right) \operatorname{Tanh}\left(\frac{x_{R}-x_{L}}{2 L_{M}(x, t)}\right) \\
Q_{R}=q A L_{R}(x, t) \sqrt{P_{R}^{2}-P_{W}^{2}} q A .
\end{gathered}
$$

Finally, the expressions to calculate $x_{L}$ and $x_{R}$ are given by (41) and (42).

$$
\begin{aligned}
& x_{L} \approx \sqrt{\frac{2 \varepsilon_{s}\left(V_{b i}+V_{j 1}\right)}{q N_{D}}} \\
& x_{R} \approx \sqrt{\frac{2 \varepsilon_{s}\left(V_{b i}+V_{j 2}\right)}{q N_{D}}} .
\end{aligned}
$$

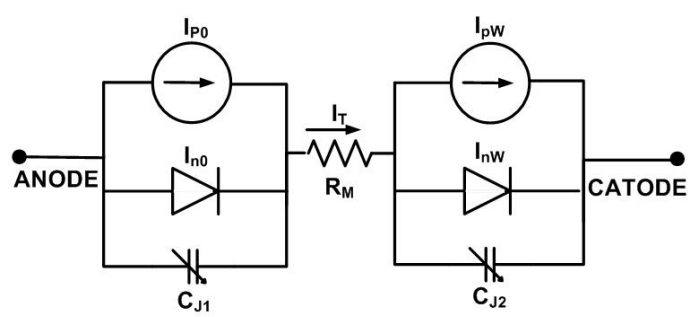

Fig. 8. Simplified electric equivalent to simulate the $\mathrm{SiC}$ power diode.

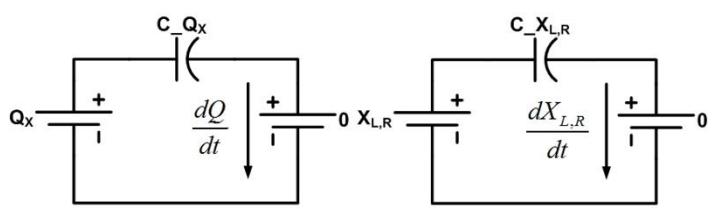

Fig. 9. Auxiliary circuits that simulate the nonlinear terms in Pspice.

\section{Model IMPLementation}

In Fig. 9, the auxiliary electrical circuits used to solve the non-nonlinear terms: $d Q_{O N, M, L, R} / d t$ and $d X_{L, R} / d t$ presented in (30)-(31) and (35)-(37), are shown. The expressions (23), (28), (31) and (33) assure an $A D E$ continuous time solution This can allow for the easy implementation of auxiliary circuits that have fewer numerical convergence problems in the simulation.

\section{SIMULATION RESULTS}

To simulate the on-state rectifier circuits shown in Figure $10 \mathrm{a}$, and for the dynamic phases, the chopper circuit shown in Figure $10 \mathrm{~b}$ was used. In order to obtain simulation results for the model developed in Pspice, the power diode structure for an $I_{D_{M A X}}=5 \mathrm{~A}$ was calculated. The main calculated parameters that were used in the implementation of the model can be seen in Table I.

In Fig. 11, the simulation results for the static behavior in the range of $25^{\circ} \mathrm{C}$ to $225^{\circ} \mathrm{C}$ are presented. The observed onvoltage presents a dependency with respect to temperature, which is indicative of an increment in the life time, and a decrement of the bandgap value. In Fig. 12, the comparison between the experimental results reported in [15] and the results of the Pspice-implemented model for the $\mathrm{SiC}$ power diode are shown.

In Fig. 13, the simulation results of the turn-on with the dependency on temperature are presented. When the temperature is increased the on-voltage decreases and the current conduction increases, which is in agreement with the theoretical behavior expected according to the on-state. In Fig. 14, the behavior for the inverse recovery in the turn-off is shown. The conditions previous to the commutation are $\mathrm{ID}=5 \mathrm{~A}$ and $V_{O N}=2.7 \mathrm{~V}$. As was observed in the simulation, when the temperature increases, the on-voltage decreases and the inverse current peak increases.

TABLE I

MAIN PARAMETERS FOR SIC USED IN SIMULATION

\begin{tabular}{|l|c|c|}
\hline \multicolumn{1}{|c|}{ Parameter } & Symbol & Value \\
\hline Active area & $A$ & $0.04 \mathrm{~cm} 2$ \\
\hline Low doping region width & $W_{B}$ & $37 \mu \mathrm{m}$ \\
\hline Life time (300K) & $\tau_{n}=\tau_{p}$ & $0.15 \mu \mathrm{S}$ \\
\hline Electrons mobility (300K) & $\mu_{n}$ & $947 \mathrm{~cm} 2 / \mathrm{V} . \mathrm{s}$ \\
\hline Holes mobility (300K) & $\mu_{p}$ & $108 \mathrm{~cm} 2 / \mathrm{V} . \mathrm{s}$ \\
\hline
\end{tabular}




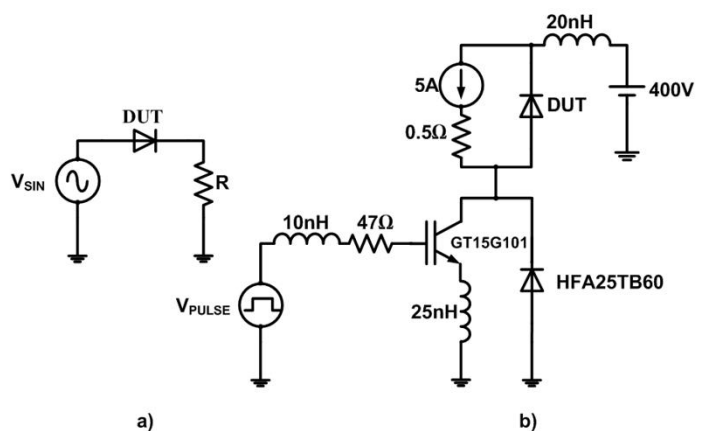

Fig. 10. Test circuit to simulate transient characteristics.

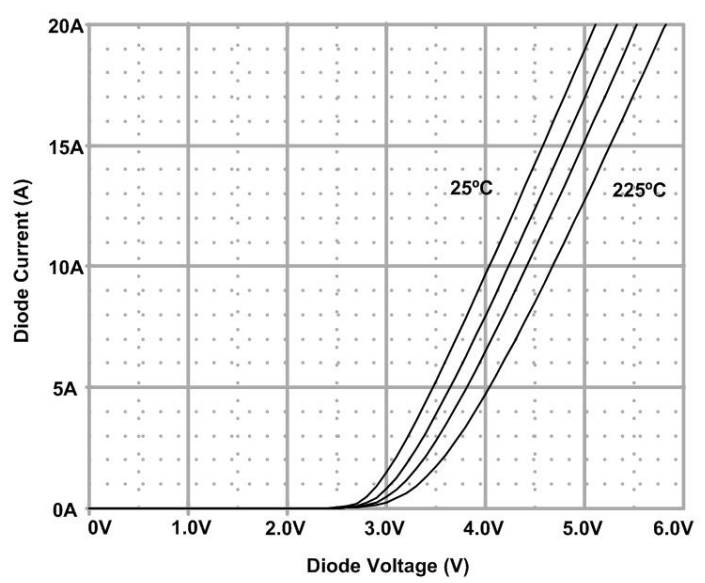

Fig. 11. Simulation result for the on-state of the power diode @ 5A.

In Fig. 15, a comparison between the experimental results reported in [15] and the results for the turn-off of the Pspiceimplemented model for the $\mathrm{SiC}$ power diode are shown. As can be seen in this comparison, the model predicts the reverse recovery behavior accurately. Finally, Figure 16 shows the behavior of the resistor in the depletion regions (Rd) and the resistor in the low doping region (Rld). Once the turn-off phase begins $(t=323.08 \mathrm{~ns})$ the resistor in the low doping region decreases its value to the order of $\mathrm{m} \Omega$ and the resistor in the depletion regions increase its values to the order of $\mathrm{M} \Omega$.

\section{CONCLUSIONS}

A methodology to solve the $A D E$ from an empirical approximation based on calculating the ambipolar diffusion length, has been proposed. The model proposed in this paper has interesting advantages with respect to other models proposed in the literature, where the accuracy of the simulation results is highly related to the number of parameters used in the model. Simulation results in Pspice have been presented which allow validating the injected charges into the $N$ - region and they have been corroborated with the theoretically expected values. The model was simulated for a maximum conduction current of $5 \mathrm{~A}$ in a temperature range of $25^{\circ} \mathrm{C}$ to $225^{\circ} \mathrm{C}$. The model is easy to implement and takes into account the main phenomena that appears in silicon carbide.

\section{ACKNOWLEDGMENT}

This work was supported by COFAA-SIP-I.P.N. and CONA-

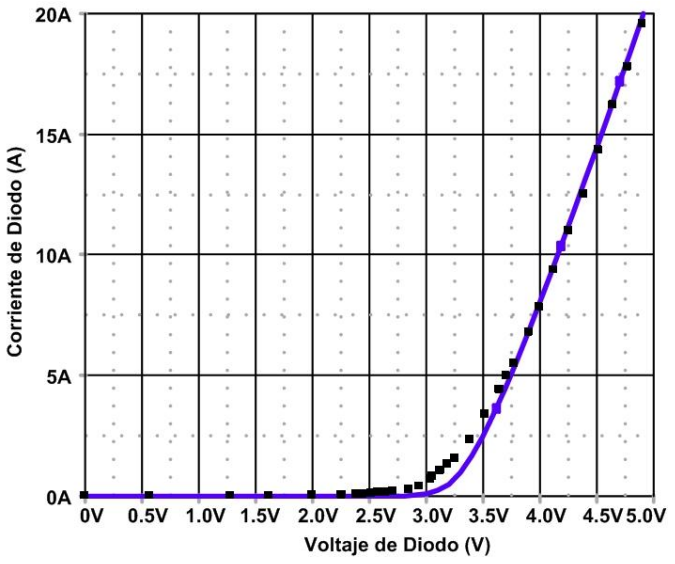

Fig. 12. Measured (Symbols) and model (Lines) on-state characteristics for, the $5 \mathrm{~A} \mathrm{SiC}$ power diode.

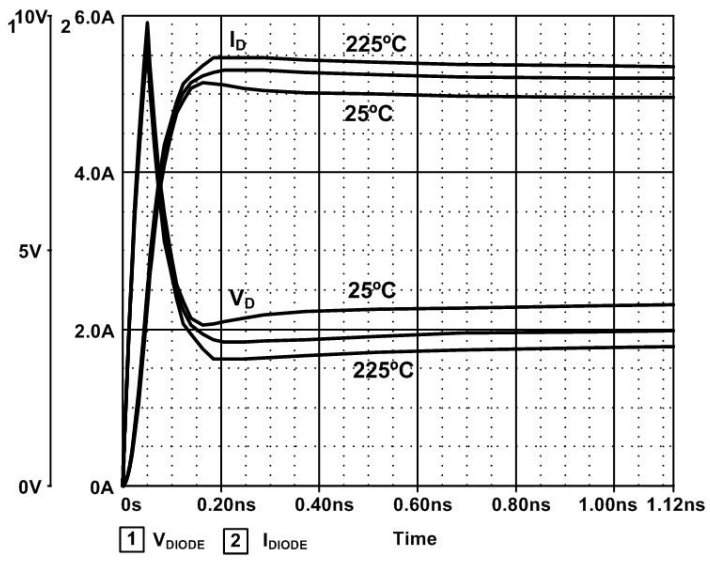

Fig. 13. Simulation for the turn-on phase, $V_{D} \approx 2.7 \mathrm{~V}$ and $I_{D} \approx 5 \mathrm{~A}$.

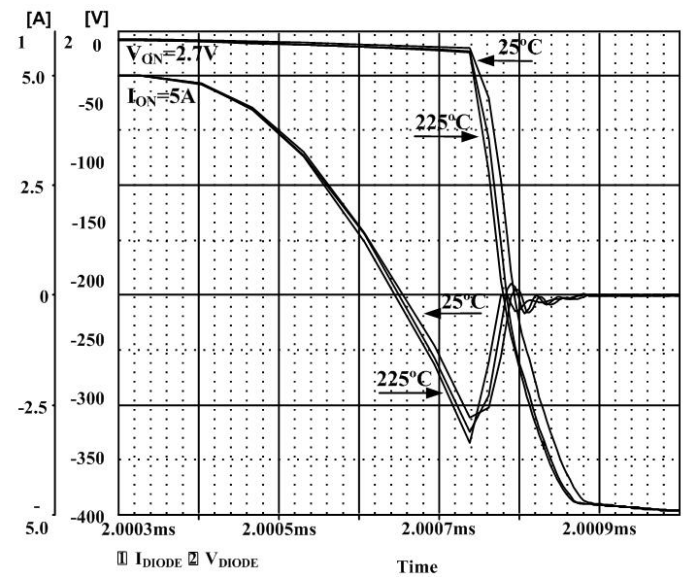

Fig. 14. Waveforms for the reverse recovery of the SiC power diode with $I_{D}=5 \mathrm{~A}$. CYT. 


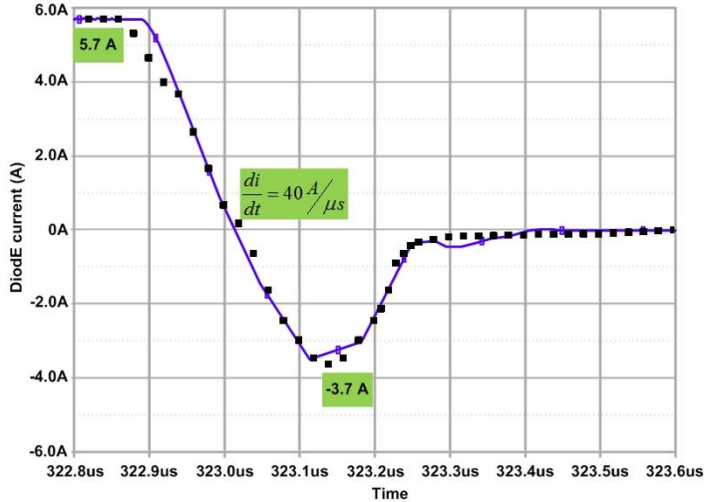

Fig. 15. Measured (Symbols) and model (lines) reverse recovery waveforms of the, 5.7A SiC power diode for a $d i / d t=40 \mathrm{~A} / \mu \mathrm{s}$.

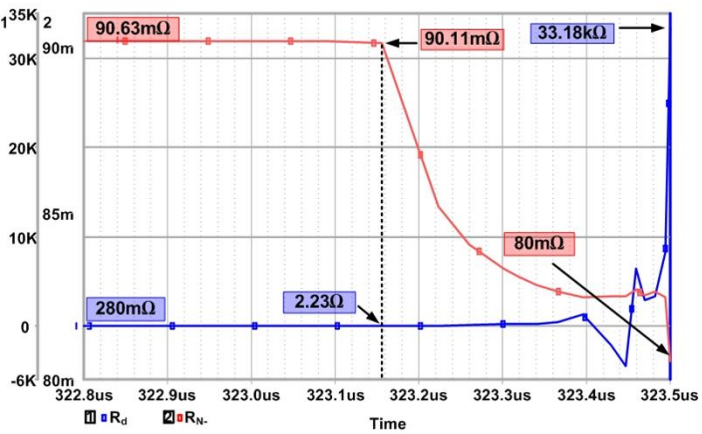

Fig. 16. Behavior of the resistors in the depletion (Rd) and low doping regions (Rld).

\section{REFERENCES}

[1] J. L. Hudgins, G. S. Simin, E. Santi, and M. A. Khan, "An assessment of wide bandgap semiconductors for power devices," IEEE Trans. Power Electron., Vol. 18, No.3, pp. 907-914, May 2003.

[2] M. E. Levinshteina, P. A. Ivanova, M. S. Boltovetsb, V. A. Krivutsab, J. W. Palmourc, M. K. Dasc, and Brett A. Hullc, "High-temperature (up to $773 \mathrm{~K}$ ) operation of 6-kV 4H-SiC junction diodes," Solid-State Electronics, Vol. 49, No.7, pp. 1228-1232, Jul. 2005.

[3] M. Holza, G. Hultschb, T. Scherga, and R. Ruppa, "Reliability considerations for recent Infineon SiC diode releases," Microelectronics Reliability, Vol. 47, No. 9-11, pp. 1741-1745, Sep./Nov. 2007.

[4] R. Singh, S.-H. Ryu, D. C. Capell, and J. W. Palmour, "High temperature $\mathrm{SiC}$ trench gate p-IGBTs," IEEE Trans. Electron Devices, Vol. 50, No. 3, pp. 774-784, May 2003.

[5] J. H. Zhao, L. Fursin, L. Jiao, X. Li, and T. Burke, "Demonstration of $1789 \mathrm{~V}, 6.68 \mathrm{~m} \Omega$-cm $24 \mathrm{H}-\mathrm{SiC}$ merged- PiN-Schottky diodes," Electron Devices Letters, Vol. 40, No. 6, Mar. 2004.

[6] Y. Sugawara, D. Takayama, K. Asano, A. Agarwal, S. Ryu, J. Palmour, and S. Ogata, "12.7KV Ultra High Voltage SiC Commutated Gate Turnoff Thyristor:SiCGT," Symposium on Power Semiconductor Devices \& ICs, pp. 365-368, 2004.

[7] T. Kimoto, H. Kawano, and J. Suda, " $1330 \mathrm{~V}, 67 \mathrm{~m} \Omega \mathrm{cm} 2$ 4H-SiC (0001) RESURF MOSFET," Electron Devices Letter, Vol. 26, No.9, pp. 649651, Sep. 2005

[8] P. A. Ivanova, M. E. Levinshteina, J. W. Palmourb, M. K. Dasb, and B. A. Hull, "High power $4 \mathrm{H}-\mathrm{SiC}$ P-i-N diodes (10 kV class) with record high carrier lifetime," Solid-State Electronics, Vol. 50, No. 7-8, pp. 1368-1370. Jul./Aug. 2006.

[9] S.-H. Ryu, S. Krishnaswami, B. Hull, J. Richmond, A. Agarwal, and A. Hefner, "10 kV, 5A 4H-SiC Power DMOSFET", International Symposium on Power Semiconductor Devices \& ICs, pp. 1-4, 2006.

[10] J. Zhang, P. Alexandrov, T. Burke, J. H. Zhao, "4H-SiC Power Bipolar Junction Transistor With a Very Low Specific ON-Resistance of $2.9 \mathrm{~m} \Omega$ cm2," Electron Devices Letter, Vol. 27, No. 5, pp. 368-370, May 2006.
[11] M. Su, K. Sheng, Y. Li, Y. Zhang, J. Wu, J. H. Zhao, J. Zhang, and L. X. Li, "430-V 12.4-m $\Omega \mathrm{cm} 2$ Normally OFF 4H-SiC Lateral JFET," Electron Devices Letter, Vol. 27, No. 10, pp. 834-836, Oct. 2006.

[12] R. Kraus and H. J. Mattausch, "Status and Trend of Power Semiconductor Device Models for Circuit Simulation," IEEE Transaction Power Electronics, Vol. 13, No. 6, pp. 452-465, May 1998.

[13] T. McNutt, A. Hefner, A. Mantootha, D. Berningb, and R. Singhb "Compact models for silicon carbide power devices," Solid-State Electronics, Vol. 48, No. 10-11 pp. 1757-1762, Oct./Nov. 2004.

[14] M. E. Levinshtein , T. T. Mnatsakanov, P. A. Ivanov, R. Singh, J. W. Palmour, and S. N. Yurkov "Steady-state and transient characteristics of $10 \mathrm{kV} 4 \mathrm{H}-\mathrm{SiC}$ diodes", Solid-State Electronics, Vol. 48, No. 5, pp. 807-811, May 2004.

[15] T. R. McNutt, A. R. Hefner Jr., H. A. Mantootha, J. L. Dulierec, D. W. Berningb, and R. Singh, "Physics-based modeling and characterization for silicon carbide power diodes," Solid-State Electronics, Vol. 50, No. 3, pp. 388-398, Mar. 2006.

[16] M. Loulou, M. Abdelkrim, R. Gharbi, M. Fathallah, C. F. Pirri, E Tresso, and A. Tartaglia "Modelling and analysis of a-SiC:H p-i-n photodetectors: Effect of hydrogen dilution on dynamic model," SolidState Electronics, Vol. 51, No. 7, pp. 1067-1072, Jul. 2007.

[17] S. Sze, Physics of Semiconductor Devices, John Wiley \& Sons, 1981.

[18] D. A. Neamen; Semiconductor Physics and Devices. University of New Mexico, USA. McGraw Hill, 2003.

[19] A. S. Grove, Physics and Technology of Semiconductor Devices, Wiley, pp. 184-185, 1967.

[20] A. T. Bryant, L. Lu, E. Santi, P. R. Palmer, and J. L. Hudgins, "Physical modeling of fast $\mathrm{p}-\mathrm{i}-\mathrm{n}$ diodes with carrier lifetime zoning, part I: Device model," IEEE Transaction Power Electronics, Vol. 23, No. 1, pp. 195 196, Jan. 2008

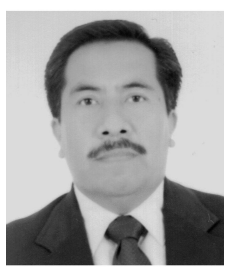

Leobardo Hernández (M’05) received his B.S. in Electronics Communications Engineering and his M.S. in Microelectronics from the National Polytechnic Institute of México City, México in 1991 and 2001, respectively. $\mathrm{He}$ received his Ph.D. in Electronics Engineering in the area of Power Electronics from the National Center for Research and Technological Development (CENIDET), Cuernavaca, Morelos, Mexico in 2009. Since 1992, he has been a full time Professor in the ESIME of the National Polytechnic Institute of Mexico. His research interests include the modelling of power devices, failure detection and characterization.

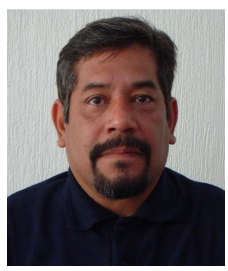

Abraham Claudio (S'96-M'00) received his B.S. and M.S. in Electrical Engineering from the Technological Institute of Laguna, México in 1983 and 1987, respectively. He received his advance studies degree (DEA) and his Ph.D. in Electrical Engineering (Power Electronics) from the National Polytechnic Institute of Grenoble (INPG), France in 1992 and 1995, respectively. Since 1987, he has been with the National Center for Research and Technological Development (CENIDET), Cuernavaca, Morelos, Mexico as a full time Professor in the Power Electronics Group. His research interests include the characterization and modelling of power semiconductor devices, AC motor control, and fault detection.

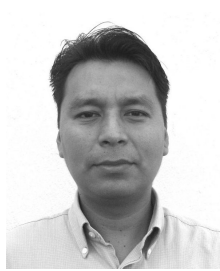

Marco A. Rodríguez (S'09-M'10) received his B.S. in Electronics Engineering from the Technological Institute of Orizaba, Veracruz, México in 1997. He received his M.S. and Ph.D. in Electronics Engineering from the National Center for Research and Technological Development (CENIDET), Cuernavaca, Morelos, Mexico in 2001 and 2009, respectively. Since 2001, he has been with the Autonomous University of Carmen City (UNACAR), Campeche, Mexico as a full time Professor in the Department of Electronics Engineering. His current research interests include electronics circuit systems, the characterization and parameters extraction of power semiconductor devices, and fault detection in motor drives. 


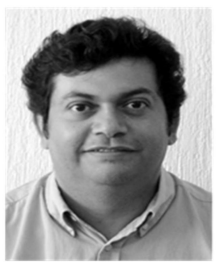

Mario Ponce was born in San Luis Potosi, S.L.P. on May 24, 1970. He received his B.E. from the San Luis Potosi Autonomous University in 1993. He received his M.S. in 1996 and his Ph.D. in 1999 from CENIDET. Since March 1999 he has been a full time Associate Professor and Researcher at CENIDET. He is Senior Member of the IEEE. He is also an AE of the IEEE Transactions on Power Electronics and he has worked as reviewer for the IEEE Transactions on Power Electronics, the IEEE Transactions on Industrial Electronics, and the IEEE Transactions on Industry Applications.

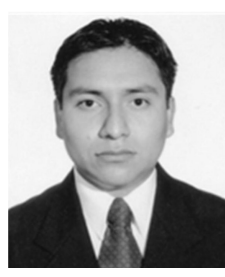

Alejandro Tapia was born in Mexico City on November 10,1986 . He received his B.S. in Communications and Electronics Engineering from the Nationa Polytechnic Institute of Mexico (IPN) in 2008. He is currently pursuing his M.S. in the Postgraduate and Research Section of the School of Mechanical and Electrical Engineering of the IPN, ESIME Culhuacan, Mexico City. His research interests include resonant converters, soft switching techniques and snubber topologies. 